\title{
Orbital Picture of Yu-Shiba-Rusinov Multiplets
}

\author{
Michael Ruby, ${ }^{1}$ Yang Peng, ${ }^{2}$ Felix von Oppen, ${ }^{2}$ Benjamin W. Heinrich, ${ }^{1}$ and Katharina J. Franke ${ }^{1}$ \\ ${ }^{1}$ Fachbereich Physik, Freie Universität Berlin, 14195 Berlin, Germany \\ ${ }^{2}$ Dahlem Center for Complex Quantum Systems and Fachbereich Physik, Freie Universität Berlin, 14195 Berlin, Germany
}

(Received 11 August 2016; published 24 October 2016)

\begin{abstract}
We investigate the nature of Yu-Shiba-Rusinov (YSR) subgap states induced by single manganese (Mn) atoms adsorbed on different surface orientations of superconducting lead $(\mathrm{Pb})$. Depending on the adsorption site, we detect a distinct number and characteristic patterns of YSR states around the Mn atoms. We suggest that the YSR states inherit their properties from the Mn $d$ levels, which are split by the surrounding crystal field. The periodicity of the long-range YSR oscillations allows us to identify a dominant coupling of the $d$ states to the outer Fermi sheet of the two-band superconductor $\mathrm{Pb}$.
\end{abstract}

DOI: 10.1103/PhysRevLett.117.186801

Local magnetic moments in metals induce potential and exchange scattering of quasiparticles. When the metal enters the superconducting state, this leads to the formation of localized bound states within the superconducting gap, referred to as Yu-Shiba-Rusinov (YSR) states [1-3]. In the simplest picture, the magnetic moment is viewed as a classical impurity spin, which is exchange coupled to itinerant electrons with an isotropic Fermi surface. Treating the exchange coupling as local and isotropic, a single particle-hole symmetric pair of YSR states is predicted within the gap whose wave functions oscillate with a wavelength $\lambda_{F}$ ( $\lambda_{F}$ being the Fermi wavelength) [3-6]. Anisotropy of the Fermi surface induces a scattering pattern reflecting the symmetry of the host lattice, as discussed theoretically and observed in recent experiments $[7,8]$.

With sufficient resolution, experiments show not only one, but several pairs of YSR resonances [9-11]. The origin of multiple YSR resonances was assigned to scattering channels with different angular momenta $(l=0,1,2, \ldots)$ $[9,12]$, or to the anisotropy splitting of the magnetic states of the adsorbate $[11,13]$. The arguments were based solely on the energetic alignment of the YSR states and not on the spatial extension and patterns of the states, which would allow one to establish a link with the orbital structure of the magnetic impurity.

Here, we address the origin of multiple YSR states by combining scanning tunneling microscopy and spectroscopy (STM and STS) experiments, which are powerful tools to map the energetic and spatial characteristics of energy levels, with a theoretical analysis.

Our study is based on $\mathrm{Mn}$ adatoms placed on a $\mathrm{Pb}$ substrate. The main advantage of this system is that the $\mathrm{Mn}$ adatoms are expected to be in the $\mathrm{Mn}^{++}$configuration with five $d$ electrons. According to Hund's rules, the Mn $d$ shell is in a ${ }^{6} S_{5 / 2}$ configuration and, hence, spherically symmetric. Thus, the ion cannot change the angular momentum of the conduction electrons in an isotropic environment and in the absence of spin-orbit coupling, which facilitates comparison between experiment and theory. The $s$-wave superconductor $\mathrm{Pb}$ is an experimentally well-studied substrate due to its high critical temperature $\left(T_{c}=7.2 \mathrm{~K}\right)$, which can be readily prepared by standard ultrahigh vacuum preparation techniques [9-11,14]. It also constitutes an appealing substrate for topologically nontrivial nanostructures [15-21]. Therefore, it would be rewarding to develop a more systematic understanding of magnetic adatom systems, reaching all the way from monomers and dimers to chains or even two-dimensional arrays. Because $\mathrm{Pb}$ possesses two disjunct Fermi surfaces, it is a two-band superconductor with two distinct gaps [22], which can be resolved in STM experiments [14]. Our study provides evidence that the YSR states in this system are predominantly associated with one of the two bands.

The experiments were carried out in a SPECS JT-STM under ultrahigh vacuum conditions at a temperature of $1.2 \mathrm{~K}$. The $\mathrm{Pb}$ single crystals were cleaned by $\mathrm{Ne}^{+}$ion sputtering $\left(900 \mathrm{eV}, 1.5 \times 10^{-4}\right.$ mbar, background pressure $<1.5 \times 10^{-9} \mathrm{mbar}$ ). Annealing to $430 \mathrm{~K}$ for $30 \mathrm{~min}$ results in clean, flat, and superconducting terraces. Spectra of the differential conductance $d I / d V$ as a function of sample bias $V$ were acquired with a standard lock-in technique at a frequency of $912 \mathrm{~Hz}$. To achieve high energy resolution, we cover etched $\mathrm{W}$ tips with $\mathrm{Pb}$ by deep indentations into the clean $\mathrm{Pb}$ surface until superconductor-superconductor tunneling spectra are measured. The use of a superconducting tip together with an elaborate grounding and rf-filtering setup yields effective energy resolutions of $\approx 60 \mu \mathrm{eV}$ at $1.2 \mathrm{~K}$. Using superconducting tips involves a convolution of the densities of states of tip and substrate, so that all subgap states $\epsilon$ appear shifted by the superconducting gap of the tip $\left(\Delta_{\text {tip }}\right)$ to an energy $\mathrm{eV}= \pm\left(\epsilon+\Delta_{\text {tip }}\right)$ [23]. $\mathrm{Mn}$ adatoms were evaporated onto the clean sample in the STM at a temperature below $15 \mathrm{~K}$, resulting in a density of $\simeq 100$ atoms per $100 \times 100 \mathrm{~nm}^{2}$.

We first deposit $\mathrm{Mn}$ atoms on the $\mathrm{Pb}(001)$ surface. All $\mathrm{Mn}$ adatoms adsorb in equivalent sites, which we call $\mathrm{Mn}_{\mathrm{Pb}(001)}$. 


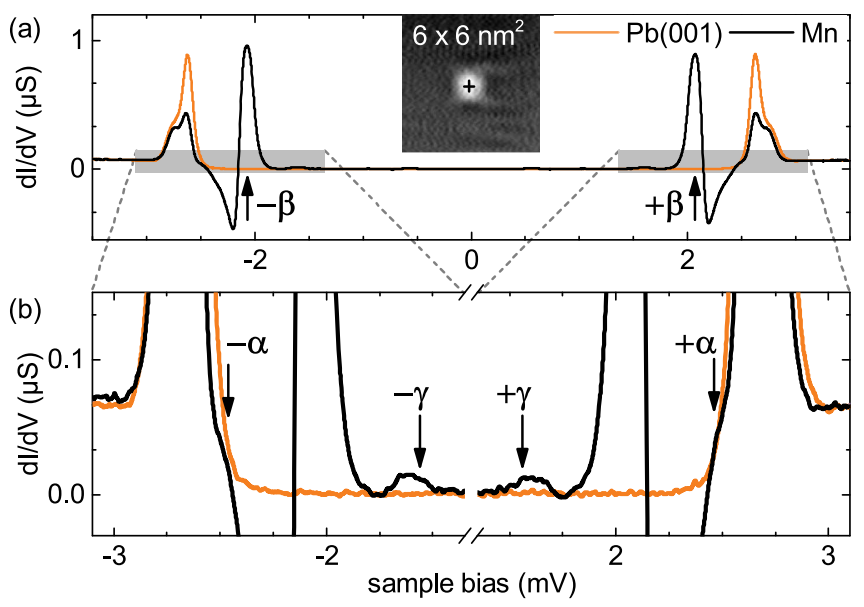

FIG. 1. (a),(b) $d I / d V$ spectrum of a Mn adatom (black) and of clean $\mathrm{Pb}(001)$ (orange). The inset shows a topography of the adatom. Three subgap resonances $\pm \alpha, \pm \beta$, and $\pm \gamma$ are marked by arrows. Set point: 300 pA, $5 \mathrm{mV}$; modulation: $15 \mu \mathrm{V}_{\text {rms }}$.

The adsorption site is stable against manipulation with the tip. In topography, the adatoms display a fourfold shape and a height of $0.15 \AA$ at $5 \mathrm{mV}$ [Fig. 1(a), inset]. Spectra on top of the adatoms reveal three pairs of YSR resonances inside the superconducting gap. The one with the largest spectral intensity (labeled $\pm \beta$ ) is found at a bias voltage of $\simeq \pm 2.08 \mathrm{mV}$ [Fig. 1(a)], complemented by two faint resonances at $\simeq \pm 2.47$ and $\simeq \pm 1.61 \mathrm{mV}$ [Fig. 1(b)]. We label the latter resonances as $\pm \alpha$, and $\pm \gamma$, respectively.

The spatial patterns of all three YSR states show characteristic fourfold symmetries [Fig. 2(b)], with extensions up to $\approx 1.6 \mathrm{~nm}$. This is an order of magnitude larger than the atomic radius. The resonances $\pm \beta$ show intensity mainly at the center of the impurity, with some weak intensity along the $\langle 110\rangle$ directions. Because of their large intensity we observe a negative differential conductance (NDC) [see Figs. 1(a) and 1(b)]. The maps at $\pm \alpha$ are dominated at the center by this NDC because of the energetic overlap of $\pm \beta$ with $\pm \alpha$; hence, the maps show no spectral intensity here; they show no spectral intensity here. The intensity is largest at a distance of $\simeq 0.9 \mathrm{~nm}$ away from the center. The map at $+\gamma$ resolves a clover leaf pattern along the $\langle 100\rangle$ directions, whereas we hardly detect any signal for $-\gamma$.

The YSR patterns resemble the shape of $d$ orbitals and thus suggest a correlation of the YSR resonances and the orbitals hosting an unpaired electron spin. This requires a splitting of the $d$ states of Mn due to the crystal field imposed by the adsorption site. In a hollow site, the nearest neighbors form a square pyramidal coordination symmetry, which removes the degeneracy of the five $d$ levels [Figs. 2(c) and 2(d)]. According to simple arguments of crystal field theory, the $d_{x^{2}-y^{2}}$-orbital lies highest, followed by the $d_{z^{2}}$ orbital, the degenerate $d_{x z}$ and $d_{y z}$ orbitals, and the $d_{x y}$ orbital at the lowest energy. The energy separation

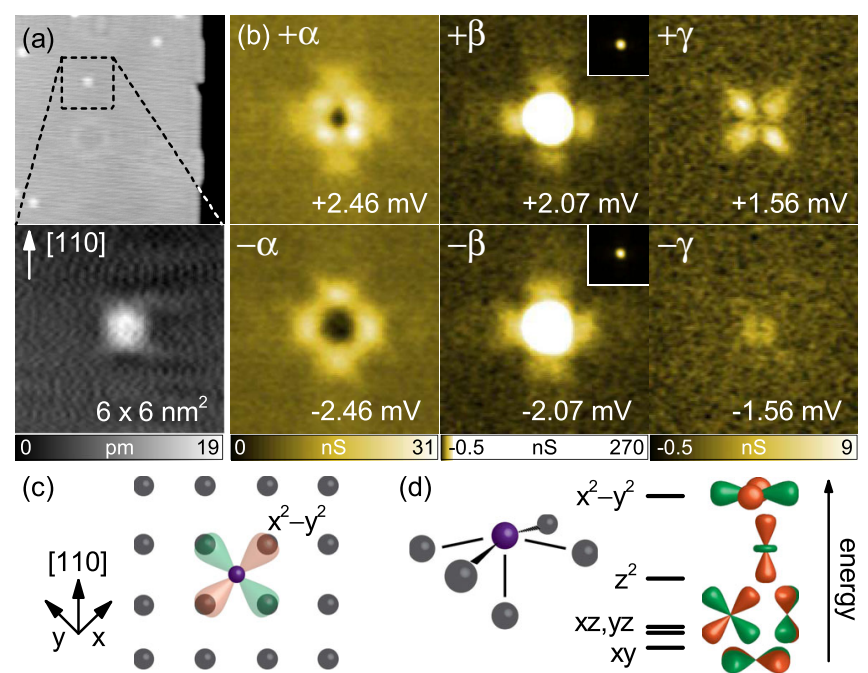

FIG. 2. (a) Topography of a Mn adatom on $\mathrm{Pb}(001)$. Set point: $50 \mathrm{mV}$ (zoom: $5 \mathrm{mV}$ ), $150 \mathrm{pA}$. (b) $d I / d V$ maps of the adatom depicted in (a) at the energy of the subgap resonances $\pm \alpha, \pm \beta$, and $\pm \gamma$ (feedback opened in each pixel at $5 \mathrm{mV}$ and $150 \mathrm{pA}$; modulation: $25 \mu \mathrm{V}_{\text {rms }}$ ). The color scale of $\pm \beta$ is stretched to enhance low intensity features (inset shows $\pm \beta$ with linear scale). The center is $\sim 100$ times more intense than the fourfold symmetric lobes. (c) Schematic top view of the adsorption of a Mn adatom in the (001) hollow site. (d) Corresponding crystal field splitting of the $d$ levels.

between $d_{x z / y z}$ and $d_{x y}$ depends on the ratio of in-plane and out-of-plane bonding distances and the levels become degenerate for an adsorption configuration with all distances being equal. Indeed, we find hints that resonance $\pm \gamma$ is composed of almost degenerate states, as it splits up upon interaction with neighboring atoms (see Supplemental Material [24]).

Simple models of YSR states rely on scattering of $l=0$ conduction electrons (for a notable exception, see Ref. [27]). However, as emphasized by Schrieffer [28], only $l=2$ conduction electrons are (potential and exchange) scattered by $\mathrm{Mn}^{++}$impurities in an isotropic metal, which is a consequence of their $S$-state nature. Starting with the isotropic case, we can then account for lattice and surface effects by the addition of anisotropic crystal fields which (partially) remove the degeneracy between the $d$ levels and make the potential and exchange coupling with the impurity orbital dependent [27] (as follows from a standard Schrieffer-Wolff transformation $[28,29])$. This structure is then inherited by the YSR states (see Supplemental Material [24]). This picture suggests that $\mathrm{Mn}^{++}$impurities actually induce five pairs of YSR states whose degeneracies and spatial patterns reflect the crystalfield-split $d$ orbitals.

We now aim for an identification of the specific $d$ orbitals that give rise to the YSR resonances $\pm \alpha, \pm \beta$, and $\pm \gamma$. The fact that $\beta$ is the most intense resonance indicates that it has the largest wave function overlap with the tip 

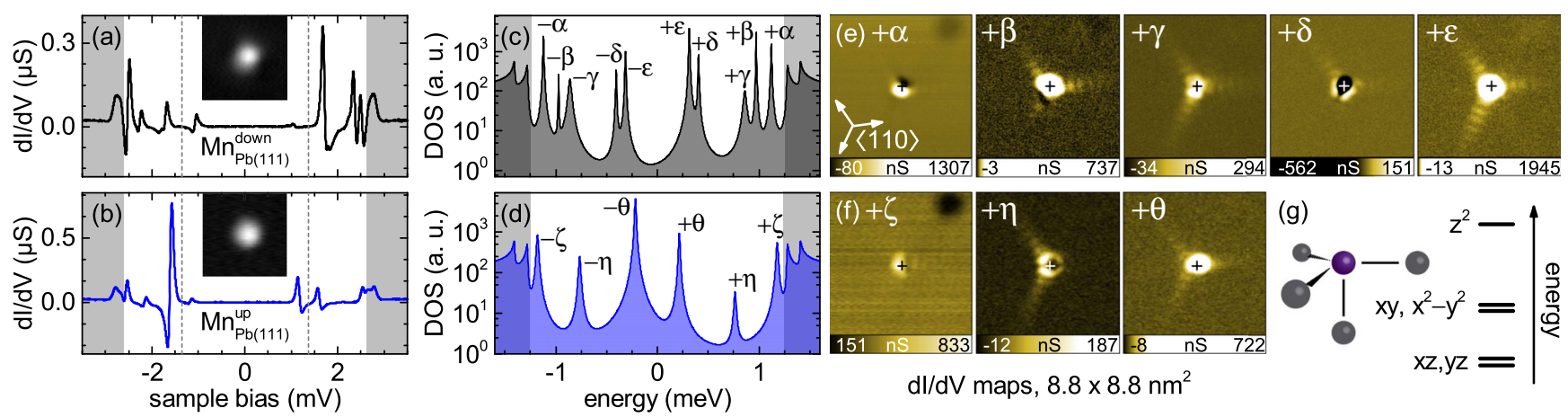

FIG. 3. (a),(b) $d I / d V$ spectra of the same Mn adatom on $\mathrm{Pb}(111)$ in the two adsorption sites as indicated in the figure. The shaded areas mark the two BCS coherence peaks [14] and the normal state. The dashed lines indicate the tip gap ( $\pm 1.38 \mathrm{mV})$. Set point: $100 \mathrm{pA}, 5 \mathrm{mV}$; lock-in modulation: $15 \mu \mathrm{V}_{\text {rms }}$. The insets show topographies of the adatom in the respective adsorption site $\left(2.6 \times 2.6 \mathrm{~nm}^{2}\right)$. (c) Deconvolved sample density of states of $\mathrm{Mn}_{\mathrm{Pb}(111)}^{\text {down }}$ exhibits five YSR resonances $( \pm \alpha, \ldots, \pm \varepsilon)$. (d) Deconvolved density of states of $\mathrm{Mn}_{\mathrm{Pb}(111)}^{\text {up }}$ shows three YSR resonances $( \pm \zeta, \pm \eta, \pm \theta)$. (e),(f) $d I / d V$ maps of the YSR resonances in both adsorption sites (feedback opened in each pixel at $5 \mathrm{mV}$ and $400 \mathrm{pA}$; modulation: $20 \mu \mathrm{V}_{\mathrm{rms}}$ ). Crosses denote the same position in all maps. The dark spot in the top right corner is a subsurface neon inclusion. The color scale is stretched to give the best contrast to spatially extended features (for maps with linear color scale, see Supplemental Material [24]). (g) Crystal field splitting of the $d$ levels for an adatom in a hollow site.

[note the color scale in Fig. 2(b)]. Moreover, the main intensity is spherically symmetric [inset of Fig. 2(b)]. Both arguments suggest that $\beta$ originates from scattering at the $d_{z^{2}}$ orbital, which is oriented along the surface normal. We note that both resonance $\alpha$ and $\gamma$ exhibit the largest intensity along the $\langle 100\rangle$ directions, i.e., towards the nearest neighbors. Hence, an assignment solely based on directions is not a priori possible. Instead, we rely on the above mentioned observation of the degeneracy of $\pm \gamma$. It includes the $d_{x z, y z}$ and $d_{x y}$ orbitals as scattering centers, whereby the latter is oriented in plane and thus only contributes weakly to tunneling. Then, resonance $\alpha$ is induced by scattering from the $d_{x^{2}-y^{2}}$ orbital.

In addition to the influence of the orbital symmetry, the long-range scattering pattern obtains structure from the anisotropy of the Fermi surface [7]. In case of $\mathrm{Pb}(001)$, the projected Fermi surface obeys a $C_{4}$ symmetry. Electron (hole) propagation along the $\langle 110\rangle$ directions appears enhanced due to focusing perpendicular to the low-curvature regions of the Fermi surface [14,30,31]. The anisotropy of the Fermi surface thus amplifies the $C_{4 \mathrm{v}}$ angular dependence of the $d$ orbitals. This imprints a faint fourfold shape on the YSR patterns. In the case of the $d_{z^{2}}$ orbital $( \pm \beta)$ it is $\sim 100$ times smaller than the spherically symmetric central part of the resonance [compare Fig. 2(b) with insets].

In order to test the validity of our model, we carried out similar experiments on $\mathrm{Pb}(111)$. This surface imposes a different crystal field on the adsorbate so that we expect different characteristic YSR energies and patterns. Deposition of $\mathrm{Mn}$ on $\mathrm{Pb}(111)$ leads to a unique adsorption site for all adatoms. In topography, they appear with a height of $\approx 0.5 \AA$ at $50 \mathrm{mV}$ and a slightly oval shape along one of the three $\langle 110\rangle$ directions [see inset in Fig. 3(a)].
By approaching the STM tip on top of a Mn adatom at $V=+5 \mathrm{mV}$ until contact formation, the atom is transferred from the initial adsorption site to a site with a larger apparent height $(\approx 1.1 \AA$ at $50 \mathrm{mV})$ and a fully symmetric appearance in topography [10]. The initial adsorption configuration is recovered by contact formation at $V=-180 \mathrm{mV}$, which yields the original height and shape, with the oval shape being oriented along one of the three $\langle 110\rangle$ directions, though not necessarily the initial one. We refer to the two adsorption sites according to their apparent heights as $\mathrm{Mn}_{\mathrm{Pb}(111)}^{\text {down }}$ and $\mathrm{Mn}_{\mathrm{Pb}(111)}^{\text {up }}$, respectively.

Both adsorption sites show several YSR resonances inside the superconducting energy gap at $\mathrm{eV}= \pm\left(\epsilon+\Delta_{\text {tip }}\right)$ [Figs. 3(a) and 3(b)]. [In addition, we also observe resonances at $\mathrm{eV}= \pm\left(\Delta_{\text {tip }}-\epsilon\right)$ which originate from thermally activated tunneling into or out of YSR states [10] and are restricted to small $\epsilon$ at $1.2 \mathrm{~K}$.] The deconvolved density of states is plotted in Figs. 3(c) and 3(d) [32]. Interestingly, we observe different numbers of YSR resonances for the two adsoption sites, in addition to shifts in energy. For $\mathrm{Mn}_{\mathrm{Pb}(111)}^{\mathrm{down}}$ adatoms, we resolve five YSR resonances, independent of the direction of the oval appearance. In contrast, $\mathrm{Mn}_{\mathrm{Pb}(111)}^{\text {up }}$ adatoms exhibit only three resonances.

The multiplicity of the YSR states is consistent with certain adsorption sites. The threefold multiplicity of the $\mathrm{Mn}_{\mathrm{Pb}(111)}^{\mathrm{up}}$ adsorption site agrees with a hollow site, which is subject to a trigonal pyramidal crystal field. This induces a $d$-level splitting with the $d_{z^{2}}$ orbital lying highest in energy, followed by the degenerate $d_{x y}$ and $d_{x^{2}-y^{2}}$ orbitals. Lowest in energy are the degenerate $d_{x z}$ and $d_{y z}$ orbitals [Fig. 3(g)]. The fivefold multiplicity of the YSR resonances of $\mathrm{Mn}_{\mathrm{Pb}(111)}^{\text {down }}$ indicates the removal of all degeneracies of 
the $d$ orbitals. This is the case when the atom is slightly displaced from a hollow site, which is consistent with its oval-shaped appearance.

Next, we investigate the spatial distribution of the YSR resonances by $d I / d V$ maps at the respective energies. They are shown for positive bias voltages in Fig. 3(e) for $\mathrm{Mn}_{\mathrm{Pb}(111)}^{\text {down }}$ and in Fig. 3(f) for the same atom after manipulation into the $\mathrm{Mn}_{\mathrm{Pb}(111)}^{\mathrm{up}}$ adsorption state. Maps at negative bias voltages reveal similar patterns (see Supplemental Material [24]). The maps do not reflect the typical fourfold shape of the Mn $d$ orbitals. The $C_{3 \mathrm{v}}$ symmetry of the ligand field polarizes the $d$ orbitals due to hybridization with the $p$ orbitals [33]. As a result, the characteristic $d$-orbital shapes are deformed, resulting in an overall twofold symmetry as reflected in the YSR maps. We may tentatively assign the YSR states by arguments of wave function overlap with the tip. Both $\pm \theta$ and $\pm \zeta$ show a large intensity signifying an out-of-plane extension of the wave function. The spherical symmetry of $\pm \theta$ at the impurity site suggests that it originates from scattering at the $d_{z^{2}}$ orbital. Resonances $\pm \zeta$ would thus correspond to the degenerate $d_{x z}$ and $d_{y z}$ orbitals. The in-plane $d_{x^{2}-y^{2}}$ and $d_{x y}$ orbitals possess the smallest wave function overlap and hence the lowest intensity at $\pm \eta$.

Another interesting feature is the large lateral extension of several of the YSR states. Three $(\beta, \gamma, \varepsilon)$ of the five states of the $\mathrm{Mn}_{\mathrm{Pb}(111)}^{\mathrm{down}}$ adatoms and two $(\eta, \theta)$ of the three states of $\mathrm{Mn}_{\mathrm{Pb}(111)}^{\text {up }}$ persist up to $4 \mathrm{~nm}$ away from the adsorbate [Figs. 3(e) and 3(f)]. The beamlike extension along the $\langle 110\rangle$ axes is due to the focusing of scattering electrons from the flat parts of the Fermi surface. We identify oscillating intensities within the beams. Indeed, an oscillation with $2 k_{F}$ is expected for YSR states, because their wave functions obey [3]

$$
\psi^{ \pm}(r) \propto \frac{\sin \left(k_{F} r+\delta^{ \pm}\right)}{k_{F} r} \exp \left(-\left|\sin \left(\delta^{+}-\delta^{-}\right)\right| \frac{r}{\xi}\right) .
$$

Here, $k_{F}$ is the Fermi wave vector, $\xi$ is the coherence length, and $\delta^{ \pm}$are the scattering phase shifts of the YSR states at positive and negative bias, respectively. The electron density $|\psi(r)|^{2}$ thus decays as $1 / r^{2}$. We have removed this dependence by a fit to the decaying intensity of states $\pm \theta$ and $\pm \eta$ (for details see Supplemental Material [24]). The result is plotted in Fig. 4 and highlights the oscillations. We observe up to four periods with a periodicity of $\simeq 5.8 \AA$, which should be compared to the Fermi wavelength of the substrate. $\mathrm{Pb}$ possesses two disjunct Fermi sheets [22]. One sheet is $s$-p-like and originates from the second Brillouin zone, the other one is $p$-d-like and originates from the third Brillouin zone. The peculiar band structure gives rise to two superconducting energy gaps $[14,22]$, which we also observe in the $d I / d V$ spectra. The corresponding Fermi wavelengths along the $\langle 110\rangle$ direction

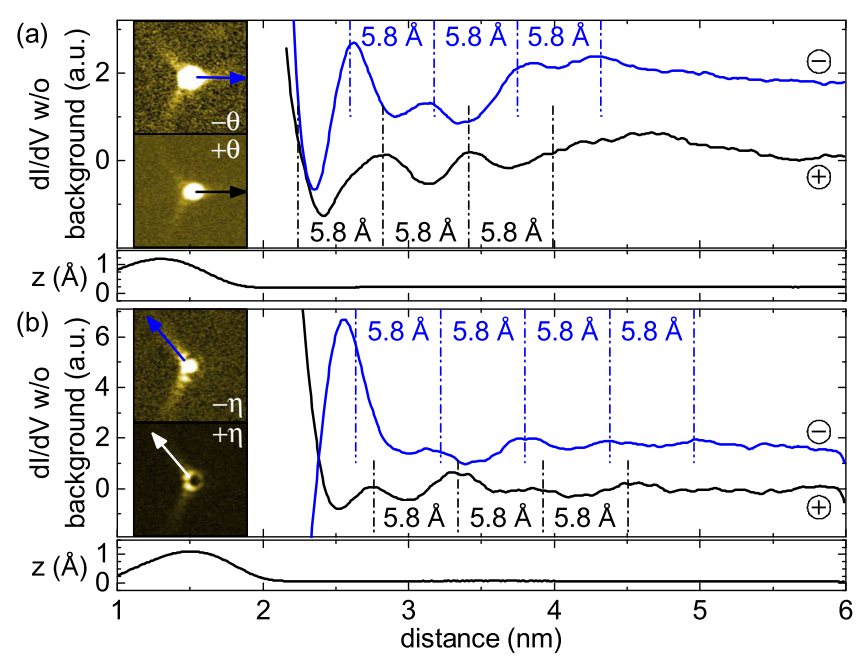

FIG. 4. Lateral evolution of the spectral intensity at positive (black) and negative bias (blue) of the two YSR resonances with lowest binding energy for $\mathrm{Mn}_{\mathrm{Pb}(111)}^{\text {up }}$. Set point: $400 \mathrm{pA}, 4 \mathrm{mV}$; modulation: $20 \mu \mathrm{V}$. The blue curve is offset by +1.5 . The $1 / r^{2}$ decay has been removed (for full data see Supplemental Material [24]). The insets show the $d I / d V$ maps at the corresponding energies. The arrows mark the direction along which the intensity is plotted. The $z$ profile gives the apparent height along the distance from the impurity center.

are $\lambda_{F}=7.8 \pm 0.8 \AA$ for the first, and $\lambda_{F}=12.1 \pm 0.5 \AA$ for the second band [34]. The observed periodicity agrees with $\lambda_{F} / 2$ of the second Fermi sheet. Hence, the YSR resonances arise due to magnetic scattering with electrons in the $p$ - $d$-like band. This is a reasonable conjecture in view of the more localized character of this band compared to the more delocalized nature of the $s$ - $p$-like band.

The oscillations of holelike and electronlike YSR resonances are phase shifted in Fig. 4 with a larger shift between resonances $\pm \theta$ compared to resonances $\pm \eta$. This agrees with the dependence of the binding energies of the YSR states on the phase shifts according to $\epsilon=\Delta \cos \left(\delta^{+}-\delta^{-}\right)$. It implies that the closer states are to the gap edge the smaller is the phase shift between the positive and negative YSR component.

To summarize, we investigated YSR states of transition metal adatoms on high symmetry surfaces of the BCS superconductor $\mathrm{Pb}$. The adsorption site imposes a distinct crystal field splitting on the $d$ orbitals. We could show that the YSR states inherit the symmetry of the scattering potential from the individual $d$ orbitals of the adatom. On the $\mathrm{Pb}(001)$ surface, spatially resolved conductance maps allow us to identify the corresponding $d$ orbitals. The strong influence of the anisotropic Fermi surface overwhelms this assignment on the $\mathrm{Pb}(111)$ surface. The oscillatory patterns reveal the Fermi wavelength of the $p$ - $d$-like Fermi sheet to be responsible for the scattering pattern. The long-range and directional nature of the states are promising for the design of coupled adatom structures. 
We acknowledge funding by the Deutsche Forschungsgemeinschaft through Grant No. FR2726/4 and through collaborative research Grants No. Sfb 658, No. CRC 183, and No. SPP 1666, as well as by the European Research Council through Consolidator Grant NanoSpin.

Note added.-Recently, we became aware of related work on $\mathrm{Cr}$ atoms on $\mathrm{Pb}(111)$ [35].

[1] L. Yu, Acta Phys. Sin. 21, 75 (1965).

[2] H. Shiba, Prog. Theor. Phys. 40, 435 (1968).

[3] A. I. Rusinov, Zh. Eksp. Teor. Fiz. Pisma Red. 9, 146 (1968) [JETP Lett. 9, 85 (1969)].

[4] A. L. Fetter, Phys. Rev. 140, A1921 (1965).

[5] A. Yazdani, B. A. Jones, C. P. Lutz, M. F. Crommie, and D. M. Eigler, Science 275, 1767 (1997).

[6] A. V. Balatsky, I. Vekhter, and J.-X. Zhu, Rev. Mod. Phys. 78, 373 (2006).

[7] M. I. Salkola, A. V. Balatsky, and J. R. Schrieffer, Phys. Rev. B 55, 12648 (1997).

[8] G. C. Ménard, S. Guissart, C. Brun, S. Pons, V. S. Stolyarov, F. Debontridder, M. V. Leclerc, E. Janod, L. Cario, D. Roditchev, P. Simon, and T. Cren, Nat. Phys. 11, 1013 (2015).

[9] S.-H. Ji, T. Zhang, Y.-S. Fu, X. Chen, X.-C. Ma, J. Li, W.-H. Duan, J.-F. Jia, and Q.-K. Xue, Phys. Rev. Lett. 100, 226801 (2008).

[10] M. Ruby, F. Pientka, Y. Peng, F. von Oppen, B. W. Heinrich, and K. J. Franke, Phys. Rev. Lett. 115, 087001 (2015).

[11] N. Hatter, B. W. Heinrich, M. Ruby, J. I. Pascual, and K. J. Franke, Nat. Commun. 6, 8988 (2015).

[12] M. E. Flatté and J. M. Byers, Phys. Rev. B 56, 11213 (1997).

[13] R. Zitko, O. Bodensiek, and T. Pruschke, Phys. Rev. B 83, 054512 (2011).

[14] M. Ruby, B. W. Heinrich, J. I. Pascual, and K. J. Franke, Phys. Rev. Lett. 114, 157001 (2015).

[15] S. Nadj-Perge, I. K. Drozdov, J. Li, H. Chen, S. Jeon, J. Seo, A. H. MacDonald, B. A. Bernevig, and A. Yazdani, Science 346, 602 (2014).

[16] R. Pawlak, M. Kisiel, J. Klinovaja, T. Meier, S. Kawai, T. Glatzel, D. Loss, and E. Meyer, arXiv:1505.06078.
[17] M. Ruby, F. Pientka, Y. Peng, F. von Oppen, B. W. Heinrich, and K. J. Franke, Phys. Rev. Lett. 115, 197204 (2015).

[18] J. Röntynen and T. Ojanen, Phys. Rev. Lett. 114, 236803 (2015).

[19] Y. Kim, J. Zhang, E. Rossi, and R. M. Lutchyn, Phys. Rev. Lett. 114, 236804 (2015).

[20] G. C. Ménard, S. Guissart, C. Brun, M. Trif, F. Debontridder, R. T. Leriche, D. Demaille, D. Roditchev, P. Simon, and T. Cren, arXiv:1607.06353v1.

[21] J. Li, T. Neupert, Z. Wang, A. H. MacDonald, A. Yazdani, and B. A. Bernevig, Nat. Commun. 7, 12297 (2016).

[22] A. Floris, A. Sanna, S. Massidda, and E. K. U. Gross, Phys. Rev. B 75, 054508 (2007).

[23] Note that the spectra reflect the density of states of the YSR states because they are recorded in the weak tunneling regime [10].

[24] See Supplemental Material http://link.aps.org/supplemental/ 10.1103/PhysRevLett.117.186801, which includes Refs. [25,26], for a description of the theoretical analysis of orbital-dependent Yu-Shiba-Rusinov states and of the crystal field splitting, as well as additional experimental data, i.e., $d I / d V$ maps and raw data.

[25] A. M. Tsvelick and P. B. Wiegmann, Adv. Phys. 32, 453 (1983).

[26] M. S. Dresselhaus, G. Dresselhaus, and A. Jorio, Group Theory: Application to the Physics of Condensed Matter (Springer, Berlin, 2007).

[27] C. P. Moca, E. Demler, B. Jankó, and G. Zaránd, Phys. Rev. B 77, 174516 (2008).

[28] J. R. Schrieffer, J. Appl. Phys. 38, 1143 (1967).

[29] A. C. Hewson, The Kondo Problems to Heavy Fermions (Cambridge University Press, Cambridge, England, 1993).

[30] O. Kurnosikov, J. H. Nietsch, M. Sicot, H. J. M. Swagten, and B. Koopmans, Phys. Rev. Lett. 102, 066101 (2009).

[31] A. Weismann, M. Wenderoth, S. Lounis, P. Zahn, N. Quaas, R. G. Ulbrich, P. H. Dederichs, and S. Blügel, Science 323, 1190 (2009).

[32] M. Ruby, SoftwareX, doi: 10.1016/j.softx.2016.04.001 (2016).

[33] Y. Jean, Molecular Orbitals of Transition Metal Complexes (Oxford University Press, New York, 2005).

[34] G. I. Lykken, A. L. Geiger, K. S. Dy, and E. N. Mitchell, Phys. Rev. B 4, 1523 (1971).

[35] D.-J. Choi, C. Rubio-Verdú, J. de Bruijckere, M. M. Ugeda, N. Lorente, and J. I. Pascual, arXiv:1608.03752. 